\title{
Evaluating Serious Games for Foreign Language Learning: An Online Grading and Visualization Tool
}

\author{
Pinelopi Krystalli, Panagiotis Arvanitis, Panagiotis Panagiotidis \\ School of French, Aristotle University of Thessaloniki, Greece
}

\begin{abstract}
The evolution of technology and the strong belief that computer games promote learning, have contributed to the increase of educational computer games available online in the field of teaching and learning a foreign language. Therefore, there is a growing need to develop an online evaluation tool for assessing educational software of a playful character in order to facilitate teachers and autonomous players/ learners in their choices.
\end{abstract}

\section{Introduction}

The early familiarization of students with the information and communication technologies has formed the way they recruit and process information as well as the way they learn. Because of the wide spread of the web and its services, digital libraries around the world are accessible to all, and especially to young people who, as Digital Natives, have the skills to exploit the knowledge/information and create their own learning paths. However, school does not seem to adapt quickly enough to this new reality, as it becomes evident that the gap between the youth's digital culture and school culture is growing everyday [1].

This gap, as well as the fact that the school's social reality is not compatible with the reality of everyday life in a post-industrial, global and hightech society, concerns the majority of students and a growing number of teachers, who wish to integrate technologies into school and adopt better practices in class [2].

In this context, proponents of Digital Game Based Learning - (DGBL: a term coined and popularized by M. Prensky after his homonymous book) argue that this type of learning "should be considered as an alternative pedagogy adapted to new learners" [1] and that digital games "have the potential to change the landscape of education" [2] because they are "ideal media for learning at a time when children's access to ICT is proliferating and their digital media experiences are allegedly accelerating" [3].
The belief that there is a relationship between learning and playing is not innovative, of course: from Piaget to the present, the educational value of games has been variously and repeatedly demonstrated. However, this relationship is redefined as innovative digital technologies have provided the opportunity to design attractive "serious games".

Yet, given the variety and abundance of educational software freely available on the internet, the selection of the most appropriate one for a specific educational objective becomes difficult and, thus, requires careful software evaluation.

In this article, we present an online assessment tool which assesses 58 serious games for $\mathrm{F} / \mathrm{L}$ teaching and learning and aims to facilitate users language teachers and autonomous learners-, to the correct/appropriate selection of a serious game that meets their specific educational needs and goals.

\section{Evaluating a serious game for $F / L$ learning and teaching}

Educational computer games designed for learning a foreign language are considered serious games.

Zyda [4] defines a serious game as "a mental contest, played with a computer in accordance with specific rules that uses entertainment to further government or corporate training, education, health, public policy, and strategic communication objectives".

As specifically mentioned in Ludus ${ }^{1}$, serious games "have an explicit and carefully thought-out educational purpose and are not intended to be played primarily for amusement".

The evolution of technology has contributed to the increase of language learning software available on the Internet.

\footnotetext{
${ }^{1}$ Ludus is a European Commission founded project, (2009 - 2012) whose objective is 'the creation of a European network for the transfer of knowledge and dissemination of best practices in the innovative field of Serious Games', http://www.ludus-project.eu/index.html. $\quad(20$ February 2013)
} 
Thus, educators are responsible for the evaluation and selection of educational software. To this end, they have to check if the software can fulfill a list of requirements:

- the specific learning objectives defined by the curricula

- $\quad$ the specific language needs of a particular target group

- the level of language proficiency of the target audience

- the suitability towards the development of communicative language competence

- the playability/interactivity desired by the students.

However, the number of software applications available on the Internet makes the procedure of their evaluation time consuming. Therefore, there is a need to design and develop a tool for the evaluation of these serious games for F / L teaching and learning in English and French, as well as a visualization method for the presentation of the evaluation results.

\section{Methodology}

The proposed tool is based on a theoretical evaluation framework whose criteria are, at the same time, criteria for instructional games design aimed at learning / teaching a foreign language.

The model is organized around five concepts: student autonomy, motivation for learning, selfassessment and feedback, consistency between the content of the game and the educational goals (when these are clearly defined by the manufacturer) and, finally, credibility [5].

Each one of these concepts is described in detail by specific measurable criteria which are presented below.

In the first phase, 100 software applications were evaluated with the use of the above mentioned model. After a cluster analysis, these applications were distributed into three groups: weak, medium and very good.

In the second phase, only the best of these applications, namely those of the latter group, were evaluated. These 58 games, 33 in French and 25 in English, were evaluated with the tool presented in this paper.

The proposed tool classifies each of these applications in terms of user autonomy, motivation, self-assessment, feedback and transparency. Furthermore, the tool indicates what specific linguistic communicative competence can be developed through each game.

\section{Theoretical Framework}

The structure of the theoretical model underlying the proposed evaluation tool allows to clearly distinguishing the concepts around which it is articulated. This clear distinction allows the user (teacher or autonomous player / learner), to choose the right software that meets his educational goals each time.

\subsection{Student autonomy}

The concept of autonomy of a student in the procedure/process of foreign language learning contains the following:

- Autonomy is the student's ability to learn and can be acquired.

- Autonomy can be taught, and that is why the collaboration between teacher and student is considered as necessary.

- Autonomy requires responsibility on the part of the student in terms of deciding on the planning, implementation and evaluation of learning.

- It requires needs analysis, free choice of resources and access to a variety of learning tools.

- It requires constant use of the target language [6a, 6b], [7], [8], [9a, 9b].

Regarding autonomy developed through video games, Sanchez [1] states that, "autonomy results from the willingness of the learner/player to accept the challenge embedded into the game and to feel responsible for solving the problem, while remaining free to make decisions".

In order to ensure the greatest degree of autonomy that can be acquired by the player / learner through an educational computer game, eight (8) criteria that must be taken under consideration in the design of an educational computer game are included in the theoretical evaluation model:

1. definition of educational objectives,

2. definition of content,

3. definition of domains,

4. definition of level of language proficiency,

5. definition of student target group,

6. clearly defined instructions,

7. easy access to game instructions in each one of its levels,

8. referral to other sources.

\subsection{Self-assessment and feedback}

Two major factors of student autonomy are selfassessment and feedback. "Being autonomous means that the player/learner gets the opportunity to assess his/her strategy her/himself and then decide if a taken decision or a given action is relevant or not" [1].

Self-evaluation is therefore a process that allows the student to judge both the result of his effort and 
the quality of the knowledge acquired against predetermined targets.

"Assessing implies getting feedback and it is therefore clear that feedback is a crucial element, in game [1]. Feedback is an important criterion for boosting performance and enhancing learning motivation as well as a significant factor in the decision-behavior-outcome cycle [10].

According to Malone [11a] and Lepper [12], performance feedback provides an ongoing challenge and helps to maintain motivation when it is: a) clear, b) regular c) constructive and d) encouraging.

Therefore, criteria describing the notions of selfassessment and feedback focus on the existence of a system that allows player/learner to evaluate his learning in order to improve it and enhance it. The player/learner should also be given the opportunity to identify and understand his weaknesses in order to intensify his efforts to develop skills according to his personal language needs.

To this end, we describe eleven criteria, mainly focusing on the importance of score indication, to inform the player/student of his performance. In addition, a scoring scale and the appearance of a table with the highest scores are proposed, as a very important element for enhancing motivation.

More specifically, the eleven criteria relating to the self-feedback are as follows:

1. score indication in each level,

2. changing score scale,

3. final score indication,

4. final score indication in a percentage scale,

5. highest scores table,

6. final score storage,

7. total number of players having already played the game,

8. point subtraction for each wrong answer,

9. point subtraction when the player asks for additional support,

10. appearance of correct answer / solution when the player answers incorrectly,

11. feedback for right and wrong answers.

\subsection{Learning motivation}

Motivation and autonomy are interdependent concepts. Ryan \& Deci introducers of the SelfDetermination Theory (SDT) argue that, autonomy, competence and social acceptance are factors that contribute decisively to strengthening/enhancing motivation [13].

The majority of teachers of all (educational) levels wish for students who are thirsty for knowledge and learning and are willing to increase their knowledge and cognitive skills. Among the researchers in DGBL, it is a common belief that serious games enhance the motivation to learn.

The criteria that refer to motivation have been formulated on the basis of Csikszentmihalyi [14],
Malone [11a, 11b], Malone and Lepper [12] and Keller [15a, 15b] writings. These theories converge on the following: in order to provide motivation for learning, a learning environment or tool must offer challenge, stimulate learner's imagination and curiosity, provide a sense of satisfaction and control, maintain user's attention and show consistency between educational objectives and content.

These elements involve the player/student's active participation and his desire to accomplish the task, even if the completion is harder than expected.

In our proposed evaluation model we propose sixteen criteria that aim to increase learning motivation in an educational computer game environment. Parameters such as time and speed are taken into account. These criteria are as follows:

1. clearly defined final goal of the game,

2. clearly defined rules of the game,

3. graded levels of difficulty,

4. restriction of level changing,

5. limitation of time to reply,

6. variation of game speed,

7. simultaneous competition against computer or other players,

8. visual reward,

9. acoustic reward,

10. reward of access to the next level,

11. visual penalization,

12. acoustic penalization,

13. fixed reinforcement,

14. variable reinforcement,

15. satisfactory graphics quality,

16. good sound quality.

\subsection{Consistency of pedagogical goals and content}

As Tagliante [16] states, the "action-oriented approach", described in the Common European Framework of Reference for Languages (2002), is basically a task oriented approach:

"The action-oriented approach reiterated all the communicative approach theories and added the idea of "task", which should be completed in a variety of environments in which the student will become active in his social life".

Electronic educational games aimed at foreign language learning are learning tools that should propose activities to the student, which, at a later time, will allow him to perform specific tasks.

In this perspective, the determination of educational objectives and content are parameters that must be taken into account in designing an electronic educational game, since they significantly affect the effectiveness of the teaching / learning procedure of the foreign language. In addition, the proposed model suggests evaluation criteria that assess the degree of consistency between educational objectives and game content. The definition of 
educational objectives and content are a set of parameters to be taken into account when designing a computer educational game. In particular, we check whether the software propose activities that promote the following competences:

1. lexical competence,

2. grammatical competence,

3. semantic competence,

4. phonological competence,

5. orthographic competence

6. orthoepic competence,

7. sociolinguistic competence,

8. pragmatic competence,

9. sociocultural competence.

\subsection{Credibility}

The final six criteria included in our model are designed to assess the degree of credibility and transparency of the use of educational computer games.

The evaluation of an online computer game in terms of transparency is essential, as for all the information on the Internet. Information provided on the web is abundant but differs from the information we get from other sources, e.g. newspapers, magazines, books, where not only the author signs the text, but the published document is subject to editorial control. "Anyone can be a writer" on the Internet" [17].

Nowadays there are too many websites operating without oversight or editorial control over them. Therefore, there are no valid, universally accepted criteria for the posted information, which can be easily changed, stolen, or falsified at a later stage $[17,18]$.

As definition of credibility we refer to the following extract [19]: "Responsibility for the provision of information means that the information should be transparent so that it can be determined if the use is appropriate under one set of rules."

Based on Kapoun's [20] evaluation criteria such as: a) accuracy, b) authority, c) objectivity, d) timeliness and e) security, we propose the following:

1. website that hosts the game belongs to an institution/organization public or private, which states its purpose and its legal status,

2. manufacturer of the game is stated,

3. name of manufacturer,

4. contact details of the manufacturer or his website (phone or email),

5. creation date of the game is indicated,

6. last date of game's content renewal is also indicated.

\section{Development}

A basic principle for the design of our online assessment tool was the creation of a model that could host a limited number of criteria consisting of different notions as mentioned above. This demand led us towards research into certain basic parameters:

- the development of the appropriate user interface,

- the design of a database able to support a vast number of concurrent users,

Creating an online assessment tool requires integrating individual criteria as well as providing various navigational mechanisms, such as a table of contents and an index. In addition, creating statistical components must be taken into consideration.

\subsection{User Interface and Database}

A modern Content Management System (CMS) was chosen for the planning and the development of the proposed tool.

Among platforms of this type, the most promising ones are Joomla and Drupal. These systems are very popular worldwide, because:

- they are "open source" and therefore free to use,

- they have advanced and versatile options,

- $\quad$ they provide a flexible and adaptable working environment as well as extended and easy to use management tools, and

- they have been developed using the PHP programming language.

Joomla and other modern CMSs, are intended to allow the system administrator to:

- organize content,

- $\quad$ adapt the presentation / publication of content,

- $\quad$ automate management tasks, and

- manage the site's users' accounts .

Joomla platform was chosen for the realization of this online assessment tool because the number of modules currently available allows a variety of experiments which is much larger than that of Drupal.

None of the above platforms integrate components for the statistical representation and management of large set of data, making them less friendly for users.

The variety of games examined and the criteria used are supported by a database (MySQL) capable of allowing multiple users requests. A conceptual model for the database has been developed in accordance with the user's needs and our specific requirements. This database consists of a data security layer for the protection of the stored content, a data management layer $[21,22]$ as well as the storage and retrieval system [23].

In order to make the visualization of the results quicker and easier, the use of "Infographics", a technique which is widely used on the Web, has been selected.

"Infographics" or "Information graphics" are graphic visual representations of information, data or 
knowledge created with the intention of presenting complex information easily and meaningfully.

By using infographics we can improve cognition and enhance the ability to see patterns and trends. The process of creating infographics can be referred to as data visualization. Several royalties' free tools of this type are available on the web. The most popular among them are [22]:

Infogr.am (http://infogr.am): a free, web-based tool with multiple themes and an easy user interface for creating simple infographics. It has the option to create charts using real data. There are about 30 chart options like radial bar graph, scatter charts, bubble graphs, and map charts. Furthermore, infogr.am can support images and video.
- It's not support the use of real data, but it's suitable for conceptual visualizations and storytelling.

- Visual.ly (http://visual.ly): a slightly different tool from all the above, as it offers integration with social networks in order to analyze Twitter and Facebook data.

For the development of the specific tool, two pages containing infographics for English (http:// infogr.am/Online-Language-Games-for-English/) and French language (http://infogr.am/OnlineLanguage-Games-for-French) has been created, using Infogr.am ("Figure 1. English Games").

For each of these languages, infographics has been used to describe the following relations and data collected:

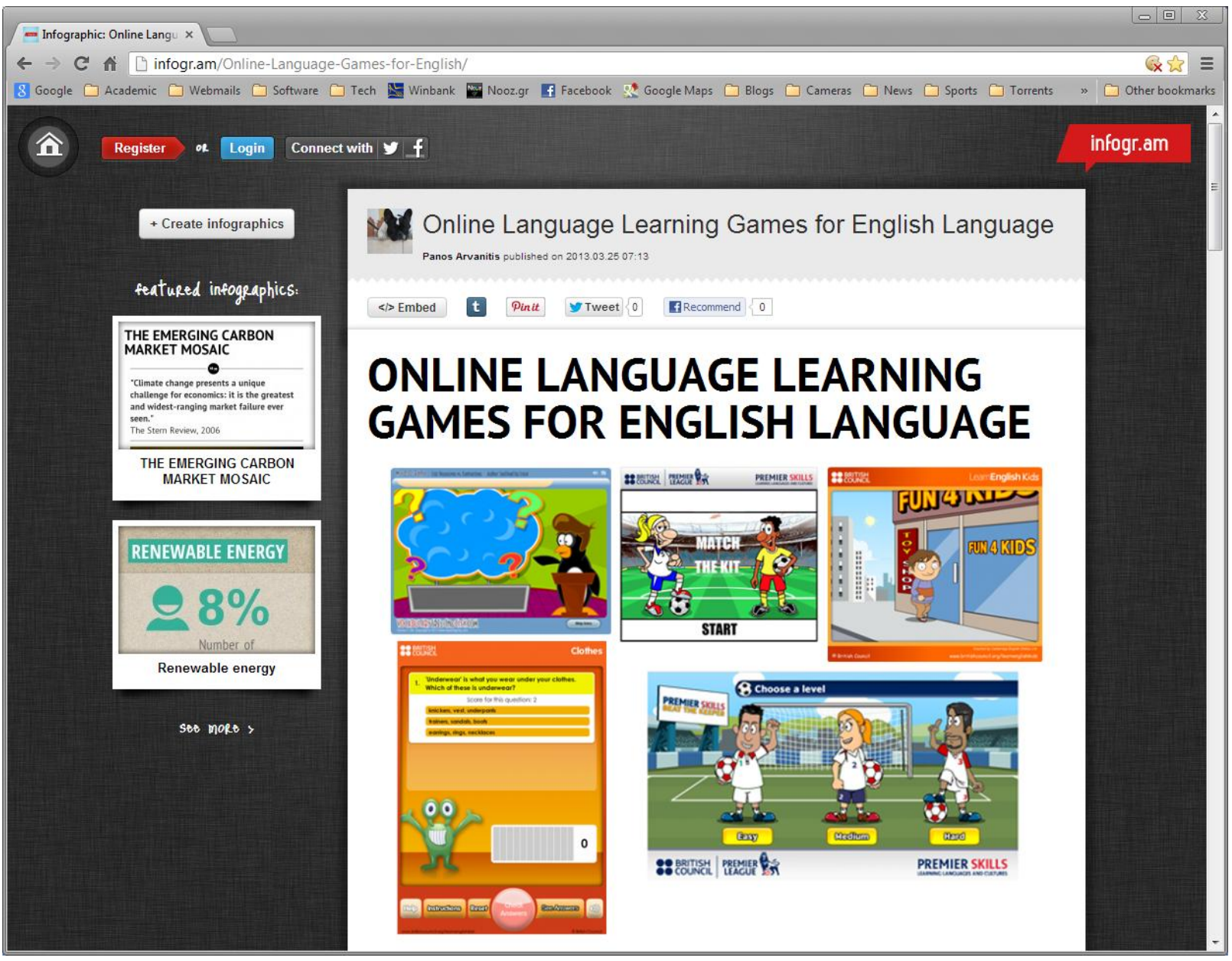

Figure 1: English games

- Piktochart (http://piktochart.com): a web-based tool for creating simple visualizations. It supports simple line, bar, and pie charts using data from a CSV file.

- Easel.ly (http://www.easel.ly): web-based tool for creating infographics.
The first infographic visualize the examined games sorted by four criteria (user autonomy, self assessment/feedback, learning motivation and credibility) ("Figure 2. Sort by Criteria").

The second infographic visualize the examined games by competence (lexical, grammatical, semantic, phonological, orthographic, orthoepic, sociolinguistic, pragmatic, sociocultural). This 
infographic also presents the score gained by fulfilling the above criteria.

- The third infographic visualize the games' titles by competence.

Using visualization via infographics, teachers have a simple and understandable guide for the evaluation of online games.

\section{Conclusion}

In an era of "digital globalization" the increasing use of open technological platforms, such as CMC, VLS, LCMS, Blogs, Wikis, social networking sites, and online gaming and simulation environments, affect the entire adulthood of young learners.

This early digital adulthood of learners, whom Prensky [24] already calls "digital natives" requires, in the field of foreign language learning, language learning tools not only suitable to their needs but capable to assist them to construct personal technology-enhanced language learning experiences. The right use of these tools by learners becomes a crucial issue for language teachers, who must guide them through the process of language acquisition.

\section{References}

[1] Sanchez, E. (2011). 'When games meet learning', in HGWE2011, http://hal.archives-ouvertes.fr/docs/00/61 /82/ 91/PDF/iigwe2011_Sanchez.pd, (12 Mars 2013)

[2] Shaffer, D.W., Squire R.K., Halverson R. and Gee J. P. (2005). 'Video Games and The Future of Learning', Phi Delta Kappan, October 2005, vol. 87, no2, pp.105-111.

[3] Williamson, B., (2008), Games and Learning Interim Report Survey of existing research and criticism. Futurlab.

[4] Zyda, M., (2005). 'From Visual Simulation to Virtual Reality to Games', Journal Computer, Vol. 38 Issue 9, IEEE Computer Society Press Los Alamitos, CA, USA, pp.25-32.

[5] Krystalli, P. (2011). An evaluation model for educational on-line computer games for foreign language teaching and learning. PHD thesis, http://invenio.lib.

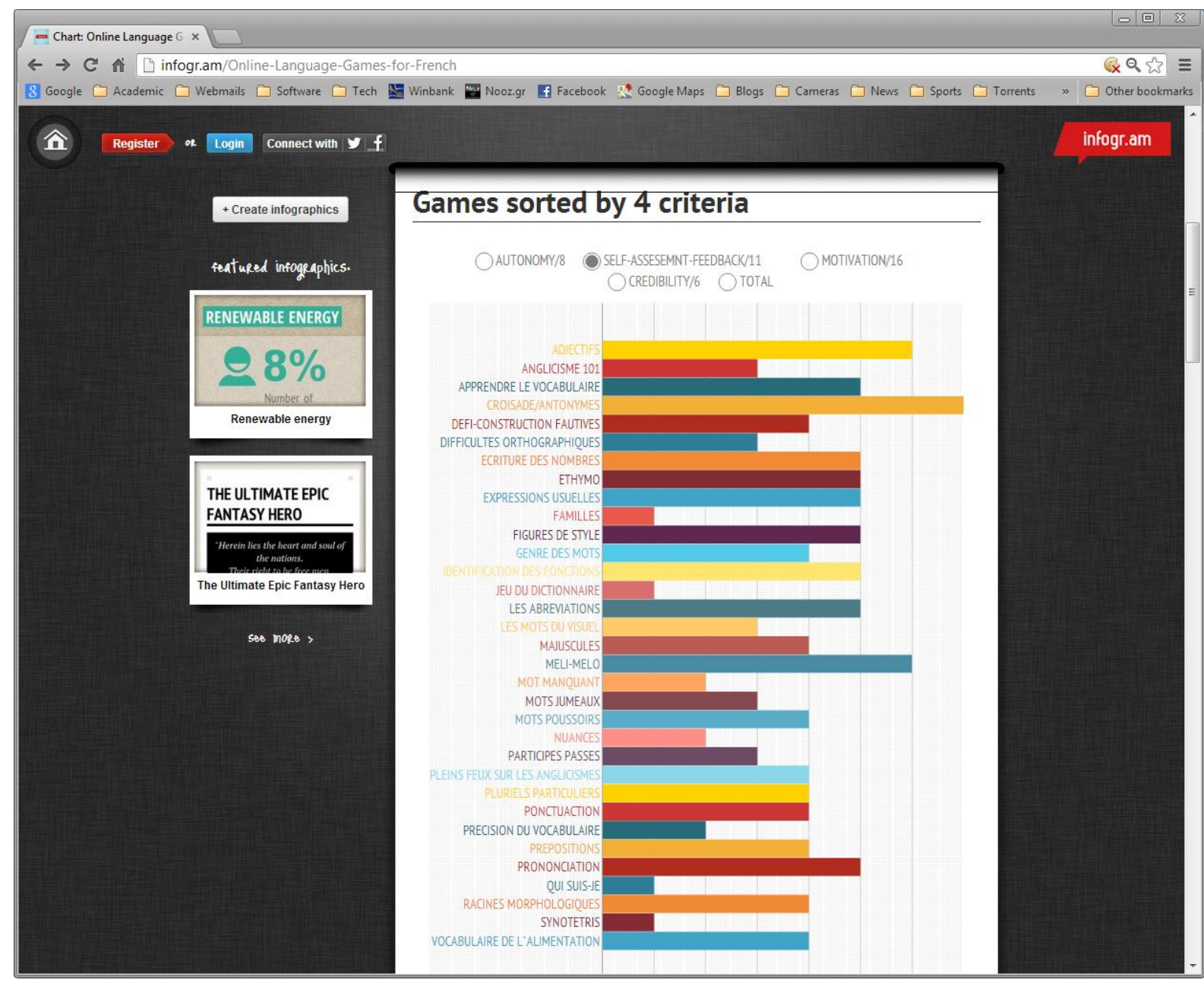

Figure 2: French games sorted by criteria 
auth.gr/ record/128661?n=el (4 November 2012).

[6a] Holec, H. (1979). Autonomie et apprentissage des langues étrangères. Hatier, Paris.

[6b] Holec, H. (1981). 'Autonomie de l'apprenant et l'apprentissage des langues', Langues Vivantes (19711981). Conseil de l'Europe, Strasbourg.

[7] Paiva, V. L. and Braga, J. F., (2008). 'The complex nature of autonomy', http://www.veramenezes.com/ autonomycomplex.pdf, (10 February 2013).

[8] Ramos, R. C., (2006). 'Considerations on the role of teacher autonomy', Colombian Applied Linguistics Journal Number 8. September 2006, pp. 183-202.

[9a] Little, D., (1991). Learner Autonomy Definitions Issues and Problems, Authentik, Dublin.

[9b] Little, D., (2004). 'Learner autonomy, teacher autonomy and the European Language Portfolio', UNTELE, University de Compiègne, 17-20 March 2004, pp.1-3.

[10] Garris R., Ahlers R., Driskell J. (2002). 'Games, Motivation, and Learning: A Research and Practice Model', Simulation and Gaming (2002), 33. pp. 441467.

[11a] Malone, T. W. (1980a). What makes things fun to learn? A study of intrinsically motivating computer games. Palo Alto, CA: Xerox.

[11b] Malone, T. W. (1980b). 'What makes things fun to learn? Heuristics for designing instructional computer games'. In Proceedings of the 3rd ACM SIGSMALL symposium, Palo Alto, California. pp. $162-169$.

[12] Lepper, M.R. \& Malone, T.W. (1987). 'Intrinsic motivation and instructional effectiveness in computerbased education'. In R.E. Snow and M. J. Farr (Eds.), 1987. Aptitude, Learning and Instruction III: Cognitive and Affective Process Analyses. Hillsdale, N.J.: Erlbaum,

[13] Ryan M. R., Deci E. L., (2000) 'Intrinsic and Extrinsic Motivations: Classic Definitions and New Directions', Contemporary Educational Psychology 25, pp.54-67, http://www.idealibrary.com, (21August 2010).

[14] Csikszentmihalyi, M. (2008). Flow-The Psychology of Optimal Experience. Harper Perennial Modern Classics, New York.

[15a] Keller, J. M. (1987). 'Strategies for stimulating the motivation to learn', Performance \& Instruction, 26 (8). pp. 1-7.

[15b] Keller, M. J. (2010). 'Five fundamental requirements for motivation and volition', Technology-Assisted Distributed Learning Environments". Inter-Ação, Goiânia, Vol. 35, No 2. pp. 305-321, http://www.revistas.ufg.br/index.php/interacao/article/dow nload (12 Mars 2013).

[16] Tagliante C, (2006), La classe de langue, CLE International, Paris.
[17] Flanagin, A. J., \& Metzger, M. J. (2000). 'Perceptions of Internet information credibility'. Journalism and Mass Communication Quarterly, 77, pp. 515-540. http://www.jasonmorrison.net/iakm/4006074.pdf (12 Mars 2013).

[18] Metzger M., Hall E. (2005). 'Understanding How Internet Users Make Sense of Credibility: A Review of the State of Our Knowledge and Recommendations for Theory, Policy, and Practice', Internet Credibility \& the User: Skills. http://projects.ischool. washington.edu/ credibility/ Metzger-skills.pdf (4 November 2012).

[19] Weitzner D.J, Abelson H, Berners-Lee T., Feigenbaum J., Hendler J., Jay Sussman G. (2008). 'Information accountability', Communications of the ACM, June 2008, Vol. 51, No. 6, http://dig.csail.mit.edu/2008/06/info-accountability-cacmweitzner.pdf pp. 83-87, (4 November 2012).

[20] Kapoun, Jim. (1998). 'Teaching Undergrads WEB Evaluation: A Guide for Library Instruction', $C \& R L$ News (July/August 1998). pp. 522-523.

http://olinuris.library.cornell.edu/ref/research/webeval.html (4 November 2012).

[21] Elmasri, Ramez, and Navathe, Shamkant B. (2000) Fundamentals of Database Systems, 3rd edition, Massachusetts: Addison-Wesley, Reading.

[22] Date, Chris J. (1995). An Introduction to Database Systems. $6^{\text {th }}$ edition, Massachusetts: Addison-Wesley Publishing Company.

[23] Connolly, T. M., and Begg, C. E. (2005). Database Systems, A Practical Approach to Design, Implementation and Management, 4th edition, Massachusetts: Addison Wesley.

[24] Prensky M. (2001). 'Digital natives, digital immigrants', On the Horizon, MCB University Press, Vol. 9 No. 5. 